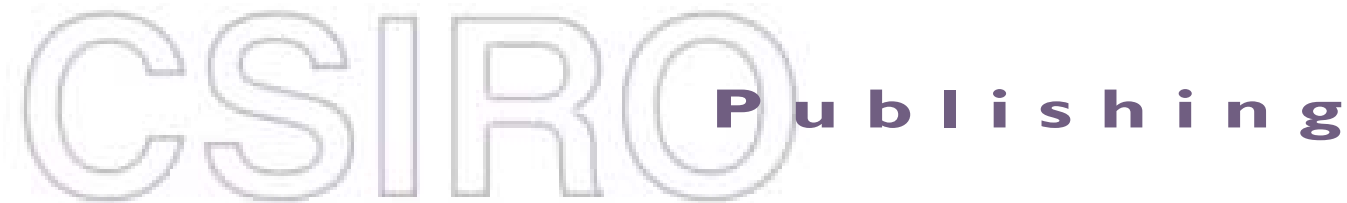

\section{Publications of the Astronomical Society of Australia}

Volume 19, 2002

(C) Astronomical Society of Australia 2002

An international journal of astronomy and astrophysics

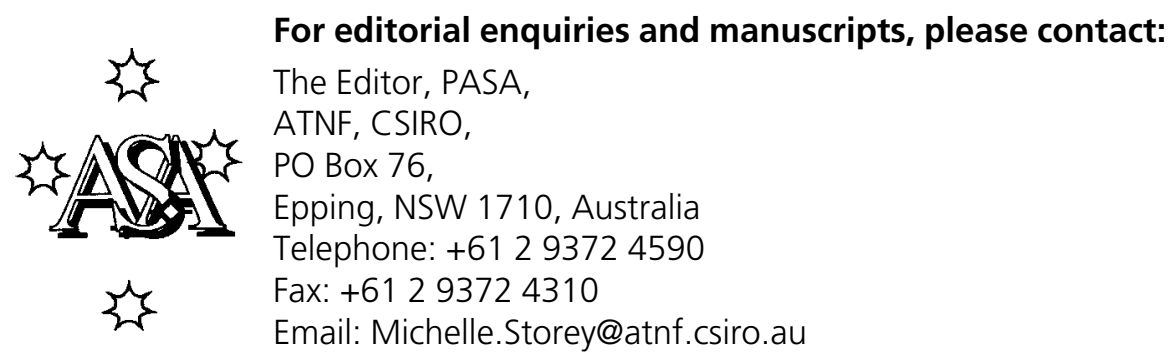

For general enquiries and subscriptions, please contact: CSIRO Publishing PO Box 1139 (150 Oxford St)

Collingwood, Vic. 3066, Australia

Telephone: +6139662 7666

Fax: +61 396627555

Email: publishing.pasa@csiro.au

C S I RO

PUBLISHING Published by CSIRO Publishing

for the Astronomical Society of Australia

www.publish.csiro.au/journals/pasa 


\title{
Molecular Masers in Variable Stars
}

\author{
Georgij M. Rudnitskij \\ Sternberg Astronomical Institute* \\ 13 Universitetskij prospekt, Moscow, 119899 Russia \\ gmr@sai.msu.ru \\ Received 2002 May 8, accepted 2002 September 17
}

\begin{abstract}
When a star with a mass of one to a few solar masses enters the red giant stage of its evolution, the radius of its atmosphere reaches several astronomical units. Pulsational instability is typical for this stage. Most stars become Mira-type or semiregular variables with light cycles of a few hundred days. Red giants lose mass at a rate $\dot{M}=10^{-7}-10^{-5} M_{\odot} \mathrm{yr}^{-1}$. Extensive gas-dust circumstellar envelopes form. These envelopes contain various molecular species. Some of these molecules $\left(\mathrm{OH}, \mathrm{H}_{2} \mathrm{O}, \mathrm{SiO}, \mathrm{HCN}\right)$ manifest themselves in maser radio emission. Data on the $\mathrm{H}_{2} \mathrm{O}$ maser variability and its connection with the stellar brightness variations are discussed. In the $\mathrm{H}_{2} \mathrm{O}$ line circumstellar masers can be divided into 'stable' (showing persistent emission - R Aql, U Her, S CrB, X Hya) and 'transient' (appearing in the $\mathrm{H}_{2} \mathrm{O}$ line once per 10-15 stellar light cycles - R Leo, R Cas, U Aur). Physical mechanisms of the maser variability are discussed. The most probable process explaining the observed visual- $\mathrm{H}_{2} \mathrm{O}$ correlation is the influence of shock waves on the masing region. Usually it is assumed that shocks in Mira atmospheres are driven by stellar pulsations. Here an alternative explanation is proposed. If a star during its main sequence life possessed a planetary system, similar to the solar system, the planets will be embedded in a rather dense and hot medium. Effects of a planet revolving around a red giant at a short distance (inside its circumstellar envelope) are discussed. A shock produced by the supersonic motion of a planet can account for the correlated variability of the $\mathrm{H} \alpha$ line emission and $\mathrm{H}_{2} \mathrm{O}$ maser. If the planetary orbit is highly eccentric, then the connected $\mathrm{H} \alpha-\mathrm{H}_{2} \mathrm{O}$ flare episodes may be explained by the periastron passage of the planet. New tasks for the upgraded ATCA are discussed.
\end{abstract}

Keywords: stars: late-type — masers — shock waves — extrasolar planets

\section{Circumstellar Molecular Masers}

At the end of its evolution, a solar-type star enters the phase of a red giant. Its radius grows from $\sim 1 R_{\odot}$ to a few $10^{2} R_{\odot}$ (Haniff, Scholz, \& Tuthill 1995). The star begins to lose matter, and an extensive gas-dust circumstellar envelope forms (Knapp et al. 1982). The red giant stage is very short as compared to the main sequence stage; it takes no more than a few hundred thousand years. The ultimate end is a complete loss of the convective shell, and formation of a naked white dwarf core surrounded by a planetary nebula (Habing 1996).

The masses of the red giants considered are from one to several solar masses, and their luminosities reach a few $10^{3} L_{\odot}$ (Clayton \& Feast 1969). Many red giants are variable; their visual brightness changes on timescales of hundreds of days. These stars are referred to as long-period variables (LPVs). The LPV subtypes are (Kholopov et al. 1985-1987):

- periodic Mira Ceti-type stars, or Miras (M), with visual brightness amplitudes $\Delta m>2.5$ magnitudes, with periods typically between 150 and 600 days;

- semiregular stars (SR); their amplitudes are $<2.5$ magnitudes, variations are less regular than in Miras and usually have shorter timescales, 50 to 150 days.

The LPV optical spectra are classified as types M, S, C. The conspicuous spectral features in them are strong

\footnotetext{
* http://www.sai.msu.su
}

molecular bands: TiO, VO in M-stars, $\mathrm{ZrO}$ in S-stars, $\mathrm{CN}$, $\mathrm{C}_{2}$ and other carbon-bearing molecules in C-stars. The set of the molecules observed reflects the chemical composition of the stars. In M-stars the $\mathrm{O} / \mathrm{C}$ atomic number ratio is greater than unity, most carbon atoms are bound in the $\mathrm{CO}$ molecules, and the remaining oxygen atoms form oxides. In $\mathrm{S}$-stars $\mathrm{O} / \mathrm{C} \sim 1$. In $\mathrm{C}$-stars $\mathrm{O} / \mathrm{C}<1$, all oxygen is in $\mathrm{CO}$, and the rest of the carbon atoms go to carbon-bearing molecules. The $\mathrm{M} \rightarrow \mathrm{S} \rightarrow \mathrm{C}$ sequence probably has an evolutionary meaning (Greene \& Wing 1975; Habing 1996). The atomic absorption lines in LPV spectra are rare, the most prominent of them is the resonant line of CaI $\lambda 4227 \AA$. Sometimes atomic emission lines appear (Balmer series of hydrogen, iron lines, etc.) - probably excited by shock waves (Wood 1979; Gillet, Maurice, \& Baade 1983; Fox, Wood, \& Dopita 1984). The stars lose matter at a rate $\dot{M} \sim 10^{-7}-10^{-5} M_{\odot} \mathrm{yr}^{-1}$ (Knapp et al. 1982). The lost matter forms a circumstellar envelope, containing molecules and dust. Circumstellar dust reradiates stellar light, producing large infrared excesses (Habing 1996).

Circumstellar molecules emit radio lines: thermal rotational lines of $\mathrm{CO}, \mathrm{SiO}, \mathrm{HCN}$ and other molecules (Olofsson et al. 1998), and maser lines $\left(\mathrm{OH}, \mathrm{H}_{2} \mathrm{O}, \mathrm{SiO}\right.$, $\mathrm{HCN})$. Circumstellar masers presently known are

- $\mathrm{OH} \lambda=18 \mathrm{~cm}(\Lambda$-doubling of the ground rotational state $^{2} \Pi_{3 / 2}, J=3 / 2: 1612,1665,1667 \mathrm{MHz}$ ) (Benson et al. 1990); 
- $\mathrm{H}_{2} \mathrm{O}$ rotational transitions: $6_{16}-5_{23} 22.2 \mathrm{GHz}$ (Benson et al. 1990), 313-2 $20183 \mathrm{GHz}$ (González-Alfonso et al. 1998), 1029-9 $36321 \mathrm{GHz}$ (Menten \& Melnick 1991), $5_{15}-4_{22} 325 \mathrm{GHz}$ (Yates, Cohen, \& Hills 1995);

- $\mathrm{SiO} \lambda=7,3.5,1.7, \ldots \mathrm{mm}$ : rotational transitions $J=$ $1-0,2-1,3-2, \ldots$ in the excited vibrational states $v=0$, $1,2,3, \ldots$ (Habing 1996);

- HCN $\lambda=3.4 \mathrm{~mm}$ : rotational transition $J=1-0$ in the excited vibrational state $v=\left(0,2^{0}, 0\right)$ (Lucas, Omont, \& Guilloteau 1988); $\lambda=1.1,0.85 \mathrm{~mm}$ : rotational transitions $J=3-2,4-3$ in the excited vibrational state $v=\left(0,1^{1 c}, 0\right)$ (Bieging, Shaked, \& Gensheimer 2000).

$\mathrm{OH}$ and $\mathrm{H}_{2} \mathrm{O}$ masers are found in oxygen-rich (M-type) stars; $\mathrm{SiO}$ masers in M- and S-type stars; $\mathrm{HCN}$ masers in carbon stars. There are a few exceptions: some C-type stars (e.g., EU And, V778 Cyg) host $\mathrm{H}_{2} \mathrm{O}$ masers (LittleMarenin, Benson, \& Dickinson 1988). Probably, they are $\mathrm{M}+\mathrm{C}$ binaries or are in a very short transition state from an oxygen-rich to a carbon-rich star.

In this paper, I give special emphasis to the $\mathrm{H}_{2} \mathrm{O}$ circumstellar masers, for which we have traced a longterm variability (see Rudnitskij et al. 2000; Lekht et al. 2001 and references therein). Furthermore, $\mathrm{H}_{2} \mathrm{O}$ masers are of interest because they trace precisely the region of the circumstellar envelope, where condensation of dust and outward acceleration of the gas-dust mixture begin (Habing 1996).

\section{Observations}

Since 1980, our team has been monitoring a sample of about 20 Miras* in the $\mathrm{H}_{2} \mathrm{O}$ maser line at $\lambda=1.35 \mathrm{~cm}$; beginning from 1994 we have also been doing optical spectroscopy of these stars (see Esipov et al. 1999 and references therein).

Extensive statistical data on the $\mathrm{H}_{2} \mathrm{O}$ maser variability of the stars suggest that there are two groups of circumstellar $\mathrm{H}_{2} \mathrm{O}$ masers.

'Stable' masers, which have been displaying $\mathrm{H}_{2} \mathrm{O}$ maser emission (however, with varying intensity) throughout our observational interval, never falling below our detection threshold ( $\sim 10 \mathrm{Jy})$. Examples are R Aql, U Her, RS Vir. For these stars the $\mathrm{H}_{2} \mathrm{O}$ maser variations correlate with the visual light curves, following them with a certain delay $\Delta \varphi$ of about $0.3-0.4 P$ ( $P$ is the brightness variation period). For U Ori this delay, analysed on a time interval of $\sim 12 P$, probably itself varies with a 'superperiod' of $\sim 9 P$ (Rudnitskij et al. 2000). A cross-correlation analysis between the visual light curve and $\mathrm{H}_{2} \mathrm{O}$ maser variations for the Mira RS Vir (Lekht et al. 2001) shows that actually the correlation is maximum for a delay between the visual and $\mathrm{H}_{2} \mathrm{O}$ light curves of 4-5P. These stars also frequently show Balmer emission lines in their spectra, in nearly every light cycle.

'Transient' masers, which sometimes disappeared from our view, falling below our detection threshold of

*http://comet.sai.msu.ru/gmr/miras.html

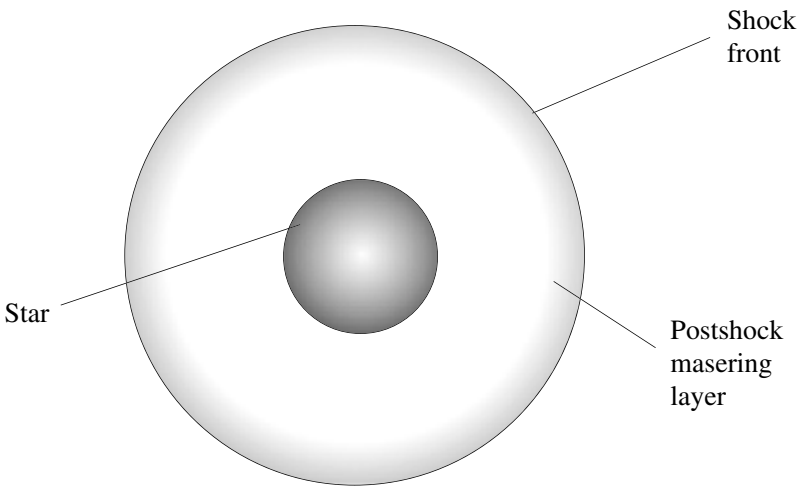

Figure 1 Model of $\mathrm{H}_{2} \mathrm{O}$ maser pumping by a spherical shock (Rudnitskij \& Chuprikov 1990).

$10 \mathrm{Jy}$ for certain intervals. Some of them (R Leo, R Cas, $\mathrm{U}$ Aur) remained ' $\mathrm{H}_{2} \mathrm{O}$-silent' for more than 15 years, but then episodically reappeared. The $\mathrm{H} \alpha$ emission was seldom observed in these stars, usually in the form of short episodes, lasting a couple of weeks, near selected light minima (Esipov et al. 1999).

In addition to this, the $\mathrm{H}_{2} \mathrm{O}$ masers from both groups display from time to time strong flares, when their $\mathrm{H}_{2} \mathrm{O}$ line flux increases a thousandfold. An example is the Mira U Ori. According to our data of March and June 1980, this star was 'silent' in $\mathrm{H}_{2} \mathrm{O}$ with the peak flux density below 10-12 Jy. However, between June and October 1980 the U Ori maser brightened to more than $1000 \mathrm{Jy}$ (Berulis et al. 1983; Rudnitskij et al. 2000). Similar events took place in the $\mathrm{H}_{2} \mathrm{O}$ maser associated with the Mira-like semiregular variable W Hya in 1981 (Berulis et al. 1983) and in 2001.

The correlation between the visual light curve and the $\mathrm{H}_{2} \mathrm{O}$ maser variations can be explained by the model of shock impact on the masing region (Rudnitskij \& Chuprikov 1990, Figure 1; see also text below). Shock waves, departing from the stellar surface under the action of pulsations, reach some time after the layers of the circumstellar envelope containing the $\mathrm{H}_{2} \mathrm{O}$ molecules. At this, collisional maser pumping is enhanced, and the maser line flux increases. The case of RS Vir suggests that this delay may be as long as a few stellar periods (Lekht et al. 2001 ), corresponding to a shock velocity of $\sim 10 \mathrm{~km} \mathrm{~s}^{-1}$.

In the star R Leo we have observed in May 1996 a short $\mathrm{H} \alpha$ line flare, followed a year and a half later by a flare in the $\mathrm{H}_{2} \mathrm{O}$ radio line (Esipov et al. 1999, Figure 2). On a smaller scale, the same sequence was observed in $\mathrm{R} \mathrm{Cas}$ and $\mathrm{U}$ Aur. All these stars were strong $\mathrm{H}_{2} \mathrm{O}$ emitters in the early 1980s, but then faded below our detection limit $(\sim 10 \mathrm{Jy})$ and remained unobservable to us until recently. The brief reappearance of $\mathrm{R}$ Leo in the $\mathrm{H}_{2} \mathrm{O}$ line 18 months after the $\mathrm{H} \alpha$ flare was the first episode of this kind we have observed. Unfortunately, there are gaps in our $\mathrm{H} \alpha$ data, and we cannot assert definitely that no similar $\mathrm{H} \alpha$ events, before or after May 1996, have been missed. However, for this particular star, strong $\mathrm{H} \alpha$ emission may be indeed an infrequent phenomenon: Castelaz \& Luttermoser (1997) 


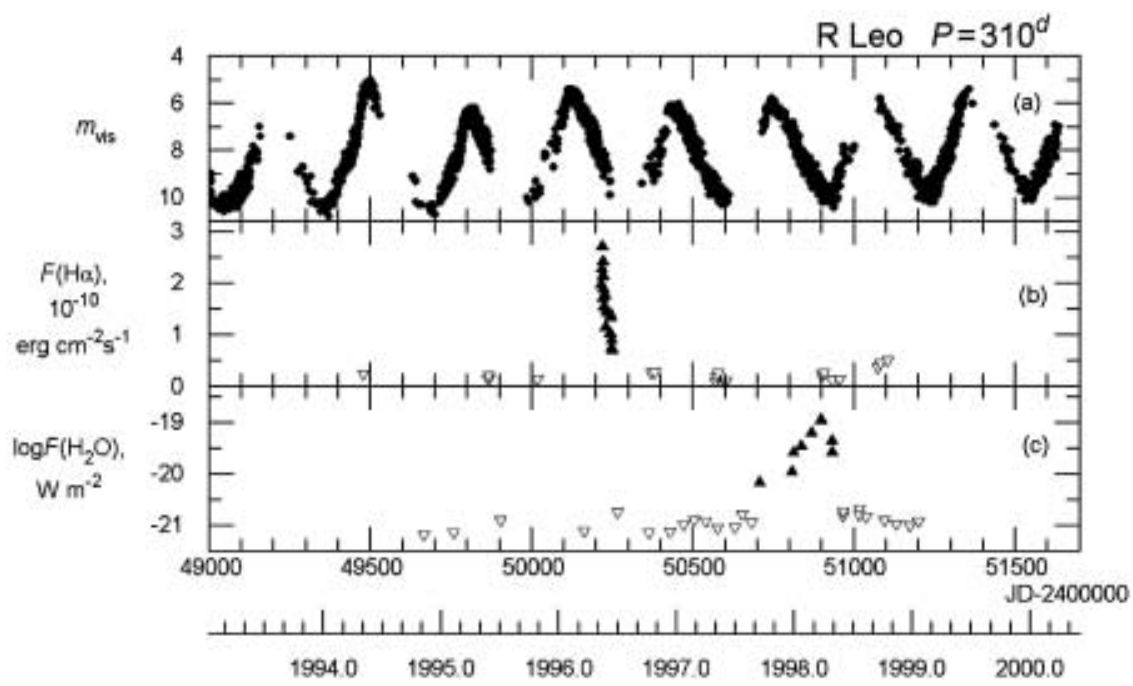

Figure 2 Observations of R Leo: (a) visual light curve (AFOEV data); (b) flux in the $\mathrm{H} \alpha$ emission line; (c) $\mathrm{H}_{2} \mathrm{O}$ line flux (Esipov et al. 1999). Open triangles: upper limits.

monitored R Leo spectroscopically at various phases of its light curve and saw a weak $\mathrm{H} \alpha$ emission only once, near light maximum. At the same time, many other stars from their sample and from ours (Esipov et al. 1999) often display rather strong $\mathrm{H} \alpha$ emission, e.g., T CrB, R Tri, $\mathrm{T}$ Her, $\mathrm{U}$ Her. This gives us reason to believe that we have really observed a rare isolated event in R Leo. Therefore, we have assumed a shock as a common cause of these two flares ( $\mathrm{H} \alpha$ and $\mathrm{H}_{2} \mathrm{O}$ flares).

If such an event is indeed rare and takes place once per 10-15 stellar light cycles, there can be various explanations. Rudnitskij \& Chuprikov (1990) explained this fact by building and disruption of a quasi-stationary layer of gas and dust (QSL), hosting the masing $\mathrm{H}_{2} \mathrm{O}$ molecules. QSL is observed in atomic and molecular absorption lines in some Miras, including R Leo, as a component at the stellar radial velocity. QSL is located at a distance of about ten stellar radii from the centre of the star; it may represent a temporary stop of the material leaving the star. In the stars studied QSL can sometimes appear and disappear (Hinkle \& Barnes 1979; Hinkle, Scharlach, \& Hall 1984). The conditions in QSL $\left(T \sim 800 \mathrm{~K}, n \sim 10^{9} \mathrm{~cm}^{-3}\right)$ are favourable for $\mathrm{H}_{2} \mathrm{O}$ maser action. The $\mathrm{H}_{2} \mathrm{O}$ maser pumping is especially enhanced if a shock wave (with a velocity of $6-10 \mathrm{~km} \mathrm{~s}^{-1}$, a stronger shock would simply dissociate molecules) crosses QSL. Behind the shock the gas is heated almost instantly, whereas some time is required to transfer heat to dust particles. Thus, there is a nonequilibrium post-shock layer with hot gas (source of energy for $\mathrm{H}_{2} \mathrm{O}$ maser pumping) and cooler dust (maser energy sink)—see Rudnitskij \& Chuprikov (1990) for details. A strong shock produces Balmer emission near the photosphere and, reaching QSL some time later, amplifies the $\mathrm{H}_{2} \mathrm{O}$ maser intensity. The passage of attenuated shocks in every light cycle of the star can explain the correlation of the $\mathrm{H}_{2} \mathrm{O}$ maser variations with the visual light curve of the star, when the $\mathrm{H}_{2} \mathrm{O}$ maser flux follows the optical brightness variations with some delay, see above. QSL can originate in a stronger mass-loss episode once per several stellar cycles. Another cause may be that pulsation-driven shocks, consecutively departing from the stellar surface, can overtake their predecessors and merge with them, thus producing, again once per several cycles, a stronger than average shock (Wood 1979), also resulting in a maser flare. If QSL vanishes for some time, the maser zone may disappear, too, and this accounts for the temporary 'silence' of the maser (as in U Ori).

The main problem may be that shocks in Miras are not so strong as has been believed. Based on the $\mathrm{H} \alpha$ emission line profiles in $o$ Cet, Gillet et al. (1983) inferred shock velocities $v_{s}$ near the photosphere of up to $90 \mathrm{~km} \mathrm{~s}^{-1}$. However, other optical spectroscopy data (Fox et al. 1984) and the weakness or lack of microwave continuum, which should accompany the H $\alpha$ emission (Reid \& Menten 1997; Chapman \& Rudnitskij 2002), suggest photospheric $v_{s}$ values no higher than $\sim 20-25 \mathrm{~km} \mathrm{~s}^{-1}$. This is difficult to reconcile with the intense Balmer emission.

As a basis of a discussion, I propose another explanation for the lack of radio continuum and for the rare $\mathrm{H} \alpha-\mathrm{H}_{2} \mathrm{O}$ episodes in the 'transient' $\mathrm{H}_{2} \mathrm{O}$ source, namely, impact of a shock provoked by a low-mass companion (a planet?) to the Mira star. This possibility is considered in the next section.

\section{Effects of a Relic Planetary System around a Red Giant}

If a star possessed a planetary system during its mainsequence life, at the red giant stage the closer planets, revolving at $R \sim 1-3 \mathrm{AU}$, will be embedded within the star's atmosphere. The more massive ones will probably survive the red giant phase (Struck-Marcell 1988; Rybicki \& Denis 2001).

Evolution of a red giant having a compact stellar companion (a brown/black dwarf with a mass of $\geq 0.02 M_{\odot}$ or possibly a neutron star) was analysed in a series of 
papers on the 'double-core evolution' (Soker 1999, and references therein). The fate of a lower-mass companion (0.001-0.01 $M_{\odot}$ - a planet), embedded in the atmosphere of a star that has become a red giant, was also considered in a number of works. In particular, Soker (1999) proposed to search for Uranus-Neptune-like planets in planetary nebulae, formed in the course of the post-AGB evolution.

Systematic observations of the last decade have led to discoveries of several tens of planets orbiting solartype stars in the solar vicinity. Most of them have been detected by the Doppler technique, involving precise measurements of stellar radial velocities on a time interval of several years, aiming to find small velocity shifts caused by the orbital motion of a low-mass companion. Depending on the planet's mass and semimajor axis, amplitudes of such velocity variations are from several $\mathrm{m} \mathrm{s}^{-1}$ to several scores of $\mathrm{m} \mathrm{s}^{-1}$. A complete list of these detections* is maintained by Jean Schneider ${ }^{\dagger}$ at the Paris-Meudon Observatory.

Of course the Doppler technique, used on a limited time interval, selects in the first instance more massive planets in orbits closer to the central stars. The planets detected so far can be divided into two groups: (1) 'hot Jupiters', planets of one to a few Jupiter masses, revolving in circular orbits very close to their stars, at $0.05-0.5 \mathrm{AU}$; (2) planets in eccentric orbits (with $e$ up to 0.6 ) with revolution periods up to a few hundred days. Certainly, further observations will find lower-mass and longer-period planets, perhaps planetary systems similar to the Solar System.

A planet orbiting around a $1 M_{\odot}$ star at a distance of $1 \mathrm{AU}$ would move at velocity $V_{p} \sim 30 \mathrm{~km} \mathrm{~s}^{-1}$. If the star is a red giant, then the planet is embedded in the star's atmosphere, having a temperature $T \sim 2000 \mathrm{~K}$ and particle number density of $\sim 10^{12}-10^{13} \mathrm{~cm}^{-3}$. The velocity of sound $a_{s}$ there would be about $3.4 \mathrm{~km} \mathrm{~s}^{-1}$. Thus, the planet's motion is supersonic, the Mach number $M=$ $V_{p} / a_{s}$ being about 9 . This motion is similar to the motion of a large meteoritic body in the Earth's atmosphere (Tsikulin 1969). A strong conical shock wave, ionising the gas and heating it to $10,000-15,000 \mathrm{~K}$, is formed.

We consider a simple model, in which a perturbing body with diameter $d$ is moving along a rectilinear trajectory at velocity $V_{p}>a_{s}$ through a medium with mass density $\rho_{0}$. Quantity $E$ is the energy released at a unit path of the body's motion; $E$ is numerically equal to the drag force exerted on the body by the medium:

$$
F=\frac{\pi d^{2}}{4} \rho_{0} V_{p}^{2}
$$

Owing to the drag, the planet is gradually spiralling into the red giant's atmosphere. The rate of decrease of its semimajor axis $a$ is (see, e.g., Taam, Bodenheimer, \&

*http://www.obspm.fr/encycl/catalog.html

${ }^{\dagger}$ http://www.obspm.fr/schneider/

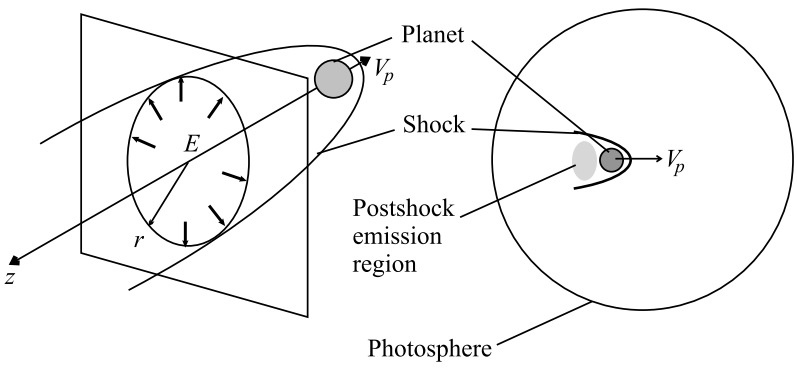

Figure 3 Left: Propagation of a shock wave within a slab perpendicular to the body's velocity vector; $E$ is the energy released in this layer. Right: Motion of a planet across the disc of a red giant at supersonic velocity $V_{p}$.

Ostriker 1978):

$$
\frac{\dot{a}}{a}=-\frac{F V_{p} a}{G M_{*} M_{p}} .
$$

Using (1) and substituting $a=1 \mathrm{AU}, M_{*}=1 M_{\odot}$, $V_{p}=30 \mathrm{~km} \mathrm{~s}^{-1}$, and Jupiter's parameters $M_{p}=1 M_{\mathrm{J}}=$ $1.9 \times 10^{30} \mathrm{~g}, d=d_{\mathrm{J}}=1.4 \times 10^{10} \mathrm{~cm}$, we have $\dot{a} / a \sim$ $-8 \times 10^{-8} \mathrm{yr}^{-1}$. Thus, during the red giant stage, which lasts not longer than $10^{6}$ years, the semimajor axis of the planet's orbit decreases by $\leq 8 \%$. For a larger planet $\left(13 M_{\mathrm{J}}, 2.35 d_{\mathrm{J}}\right)$, braking is still smaller, $\leq 3.2 \%$.

The motion of the planet through the stellar atmosphere is similar to the case of propagation of a shock wave from a detonating cylindrical charge (Tsikulin 1969). That is, in any plane perpendicular to the trajectory of the body, the propagating shock can be considered as a cylindrical one (see Figure 3). The shock front radius in this plane is

$$
r_{f}=\left(\frac{E}{\rho_{0}}\right)^{1 / 4} t^{1 / 2}
$$

with $t=z / V_{p}$. The front equation in $(r, z)$ coordinates (Figure 3, left) is

$$
\frac{r_{f}}{d}=\left(\frac{\pi}{4}\right)^{1 / 4}\left(\frac{z}{d}\right)^{1 / 2}
$$

Shock velocity $D$ in the direction perpendicular to the trajectory is decreasing with time as

$$
D=\frac{1}{2}\left(\frac{E}{\rho_{0}}\right)^{1 / 4} t^{-1 / 2} \text {. }
$$

For a planet with $d=2.35 d_{\mathrm{J}}$, velocity $D$ will fall to the velocity of sound $a_{s}$ at a distance $z_{\max }=$ $d(\pi / 4)^{1 / 2}\left(M^{2} / 4\right) \sim 5.6 \times 10^{11} \mathrm{~cm}$ behind the body. There will be no emission at greater $z$. The corresponding maximum front radius $r_{f \max }=1.3 \times 10^{11} \mathrm{~cm}$. The maximum projected area of the shocked 'cone' (for a side view, as in Figure 3, right) is

$$
\begin{aligned}
S_{\mathrm{sh}}= & \frac{1}{3}(4 \pi)^{1 / 4} d^{1 / 2} z_{\max }^{3 / 2} \sim 4.8 \times 10^{22} \mathrm{~cm}^{2} \\
& \sim 1.6 \times 10^{-5} S_{*},
\end{aligned}
$$

where $S_{*}=\pi R_{*}^{2} \sim 3 \times 10^{27} \mathrm{~cm}^{2}$ is the stellar disc area for $R_{*} \sim 3 \times 10^{13} \mathrm{~cm}$. Observations and model calculations 
of the Balmer emission lines in Miras (e.g., Fox \& Wood 1985) show that, for the above-mentioned parameters, the shock front yields up to $10^{20} \mathrm{H} \alpha$ photons $\mathrm{cm}^{-2} \mathrm{~s}^{-1}$; with source area $S_{\mathrm{sh}}$, this can account for the total Balmer line fluxes observed from a star at a distance of about $300 \mathrm{pc}$, a few $\times 10^{-12} \mathrm{erg} \mathrm{cm}^{-2} \mathrm{~s}^{-1}$ (Fox et al. 1984). The sideview observations of the cone shock also naturally explain doubling and large linewidth $\left(\sim 60 \mathrm{~km} \mathrm{~s}^{-1}\right)$ of the Balmer lines observed in Miras (e.g., Gillet et al. 1983; Udry et al. 1998). The lack of radio continuum is explained by the small angular size of the planetary source, which is thought hot enough to produce the observed Balmer emission.

Another effect of the planetary shock is enhanced $\mathrm{H}_{2} \mathrm{O}$ maser pumping by the mechanism proposed by Rudnitskij \& Chuprikov (1990); the delay may be similar to the case of a spherical shock.

As mentioned above, some stars (R Leo, R Cas and $\mathrm{U}$ Aur) displayed isolated bursts of $\mathrm{H} \alpha$ emission, followed (about a year and a half later) by a flare of the $\mathrm{H}_{2} \mathrm{O}$ maser radio emission. This may be due to a periastron shock wave episode of a planet in a highly eccentric orbit with a period $P \sim 15$ years. Some other stars (e.g., U Ori, Rudnitskij et al. 2000) have already shown some hints of $\mathrm{H}_{2} \mathrm{O}$ maser 'superperiodicity' of $12-15$ years, which could be associated with planetary revolution periods.

One of the main objections to the model proposed is that the drag of the surrounding medium tends to circularise the planet's orbit. However, Soker (2000) has shown that in the case of a mass-losing central star the passage of the periastron by a planet enhances the mass-loss rate, and this effect can support, and even increase, the orbit eccentricity.

\section{What Could Be Done on the Upgraded ATCA?}

To test the models of the circumstellar $\mathrm{H}_{2} \mathrm{O}$ maser emission proposed above, of interest would be repeated mapping of the masers. This would allow us to trace the propagation of a shock across the maser layer, igniting sequentially maser spots more and more distant from the stellar surface. Then it can be found whether the shock is spherical, as in the model of Rudnitskij \& Chuprikov (1990), or connected with a local source (a planet) moving across the stellar disc, and the shocked region trailing after it. A task for the $\mathrm{H}_{2} \mathrm{O}$ masers in C-stars (see Section 1) could be precise measurement of the maser position with respect to the optical carbon-rich star, to understand whether the $\mathrm{H}_{2} \mathrm{O}$ maser coincides with the star or is somewhat shifted and belongs to an optically obscured oxygen-rich companion. Thus it can be elucidated whether these objects are indeed binaries, consisting of carbon- and oxygen-rich stars, or stars in the transition phase from the oxygen-rich to carbon-rich state, when the photosphere already displays an optical spectrum typical of C-stars and the circumstellar envelope still contains more oxygen than carbon.

Unfortunately, such observations may be feasible only for the nearest stars (e.g., W Hya, $d \sim 90 \mathrm{pc}$ ): the angular diameter of the circumstellar $\mathrm{H}_{2} \mathrm{O}$ maser region $\left(r \sim 10^{14} \mathrm{~cm}\right)$ at a distance of $100 \mathrm{pc}$ is 70 milliarcseconds (mas), which is below the ATCA resolution at $\lambda=1.35 \mathrm{~cm}$ $\left(\sim 0.5^{\prime \prime}\right)$.

The situation is better for the shorter-wave $\mathrm{SiO}$ and $\mathrm{HCN}$ masers; here, mapping at various epochs could detect variations in the brightness and relative positions of individual $\mathrm{SiO}$ maser spots, caused by pulsation- (or planet-?) driven shocks, and distinguish the cases of a spherical shock, due to the oscillation of a star as a whole, and a 'one-sided' shock (Figure 3), appearing near the limb and travelling across the stellar disc, with $\mathrm{SiO}$ maser spots flaring behind.

Meanwhile, the method described by Plambeck, Wright, \& Carlstrom (1990) can be used. Plambeck et al. (1990) observed the $86 \mathrm{GHz} \mathrm{SiO}$ maser in Ori IRc2 on the Hat Creek millimetre-wave array with a maximum baseline of $177 \mathrm{~m}$. Measuring cross-correlation phases throughout the $\mathrm{SiO}$ spectrum, they obtained relative positions of bright Doppler features to an accuracy of 15 mas. If applied to ATCA, this method may yield relative positions of the $\mathrm{H}_{2} \mathrm{O}$ maser spots to a fraction of the ATCA beamwidth, which is to some degree a solution of the stated problem.

Continuum observations of red giants at $1.35 \mathrm{~cm}$ and $3 \mathrm{~mm}$ can be proposed, similar to those performed on the ATCA at $\lambda=6$ and $3 \mathrm{~cm}$ by Chapman \& Rudnitskij (2002). For a thermal spectrum, $F_{v} \propto v^{2}$, and shorter-wave observations are more promising for detection. In the millimetre continuum, flux densities of about a millijansky from red giants may be expected, due to photospheric emission. Episodes connected with shocks can also happen; in this case parallel monitoring in optical spectroscopy is useful for shock diagnostics.

\section{Acknowledgments}

I gratefully acknowledge the travel support from the International Astronomical Union and the MNRFIC program. I am also grateful to anonymous referees for helpful comments.

\section{References}

Benson, P. J., Little-Marenin, I. R., Woods, T. C., Attridge, J. M. Blais, K. A., Rudolph, D. B., Rubiera, M. E., \& Keefe, H. L. 1990, ApJS, 74, 911

Berulis, I. I., Lekht, E. E., Pashchenko, M. I., \& Rudnitskij, G. M 1983, SvA, 27, 179

Bieging, J. J., Shaked, S., \& Gensheimer, P. D. 2000, ApJ, 543, 897

Castelaz, M. W., \& Luttermoser, D. G. 1997, AJ, 114, 1584

Chapman, J. M., \& Rudnitskij, G. M. 2002, PASA, submitted

Clayton, M. L., \& Feast, M. W. 1969, MNRAS, 146, 411

Esipov, V. F., Pashchenko, M. I., Rudnitskij, G. M., \& Fomin, S. V. 1999, AstL, 25, 672

Fox, M. W., \& Wood, P. R. 1985, ApJ, 297, 455

Fox, M. W., Wood, P. R., \& Dopita, M. A. 1984, ApJ, 286, 337

Gillet, D., Maurice, E., \& Baade, D. 1983, A\&A, 128, 384

González-Alfonso, E., Cernicharo, J., Alcolea, J., \& Orlandi, M. A. 1998, A\&A, 334, 1016

Greene, A. E., \& Wing, R. F. 1975, ApJ, 200, 688

Habing, H. J. 1996, A\&AR, 7, 97

Haniff, C. A., Scholz, M., \& Tuthill, P. G. 1995, MNRAS, 276, 640 
Hinkle, K. H., \& Barnes, T. G. 1979, ApJ, 234, 548

Hinkle, K. H., Scharlach, W. W. G., \& Hall, D. N. B. 1984, ApJS, 56,1

Knapp, G. R., Phillips, T. G., Leighton, R. B., Lo, K. Y., Wannier, P. G., Wootten, H. A., \& Huggins, P. J. 1982, ApJ, 252, 616

Kholopov, P. N., et al. 1985-1987, General Catalogue of Variable Stars. Vols. I-III (Moscow: Nauka)

Lekht, E. E., Mendoza-Torres, J. E., Rudnitskij, G. M., \& Tolmachev, A. M. 2001, A\&A, 376, 928

Little-Marenin, I. R., Benson, P. J., \& Dickinson, D. F. 1988, ApJ, 330,828

Lucas, R., Omont, A., \& Guilloteau, S. 1988, A\&A, 194, 230

Menten, K. M., \& Melnick, G. J. 1991, ApJ, 377, 647

Olofsson, H., Lindqvist, M., Nyman, L.-Å., \& Winnberg, A. 1998, A\&A, 329, 1059

Plambeck, R. L., Wright, M. C. H., \& Carlstrom, J. E. 1990, ApJ, 348, L65
Reid, M. J., \& Menten, K. M. 1997, ApJ, 476, 327

Rudnitskij, G. M., \& Chuprikov, A. A. 1990, SvA, 34, 147

Rudnitskij, G. M., Lekht, E. E., Mendoza-Torres, J. E., Pashchenko, M. I., \& Berulis, I. I. 2000, A\&AS, 146, 385

Rybicki, K. R., \& Denis, C. 2001, Icarus, 151, 130

Soker, N. 1999, MNRAS, 306, 806

Soker, N. 2000, A\&A, 357, 557

Struck-Marcell, C. 1988, ApJ, 330, 986

Taam, R. E., Bodenheimer, P., \& Ostriker, J. P. 1978, ApJ, 222, 269

Tsikulin, M. A. 1969, Shock Waves Induced in the Atmosphere by Motion of Large Meteoritic Bodies (Moscow: Nauka) (in Russian)

Udry, S., Jorissen, A., Mayor, M., \& Van Eck, S. 1998, A\&AS, 131,25

Wood, P. R. 1979, ApJ, 227, 220

Yates, J. A., Cohen, R. J., \& Hills, R. E. 1995, MNRAS, 273, 529 